\title{
RELATIVISTIC COVARIANCE OF AN INTERACTING QUANTUM FIELD
}

\author{
BY JOHN T. CANNON ${ }^{1}$ AND ARTHUR M. JAFFE ${ }^{2}$
}

Communicated by I. M. Singer, January 23, 1970

I. Introduction. Physicists believe that quantum field theory can describe the interactions between elementary particles. The difficulties in constructing model quantum field theories become more tractable in two dimensional space-time. The best understood two dimensional model is the " $\lambda\left(\phi^{4}\right)_{2}$ quantum field theory" [1]-[4]. We discuss this model which describes a self interacting boson field $\phi(x, t)$.

Let $\leftrightarrow$ be a bounded open subset of $R^{2}$. For $(x, t) \in ß$, the field $\phi(x, t)$ is a sesquilinear form defined on a dense domain $D$ in a Hilbert space 3C. Furthermore $\phi$ is continuous in $(x, t)$ and satisfies the nonlinear partial differential equation

$$
\left\{\frac{\partial^{2}}{\partial t^{2}}-\frac{\partial^{2}}{\partial x^{2}}+m^{2}\right\} \phi+4 \lambda \phi^{3}=0 .
$$

The nonlinear term $\left(\phi^{3}\right)(x, t)$ is defined in [3], and (1) holds on $D \times D$ as an equation for Schwartz distributions.

For real $f \in \mathbb{C}_{0}^{\infty}$, the sesquilinear form

$$
\phi(f)=\int \phi(x, t) f(x, t) d x d t
$$

uniquely determines a selfadjoint operator $\phi(f)$ [3]. Let $\mathfrak{A}(B)$ denote the von Neumann algebra

$$
\mathfrak{A}(B)=\left\{e^{i \phi(f)}: f=\bar{f} \in \mathfrak{e}_{0}^{\infty}, \operatorname{supp} f \subset B\right\}^{\prime \prime} .
$$

One can interpret $\mathfrak{A}(B)$ as the bounded observables in the spacetime region $B$. It is convenient to work with the $C^{*}$-algebra $\mathfrak{A}$ of quasilocal observables defined as the norm closure of $\bigcup_{\circledast \subset R^{2} \mathfrak{P}}(B)$.

AMS Subject Classifications. Primary 8135, 8122, 8147; Secondary 8146.

Key Words and Phrases. Lorentz covariance, quantum field theory models, Poincaré automorphism, Haag-Kastler axioms, nonlinear relativistic wave equations, self interacting boson field, von Neumann algebra, $\mathrm{C}^{*}$-algebra, selfadjoint local generator.

1 Supported in part by the U. S. Air Force Office of Scientific Research, Contract No. F44620-67-C-0029.

2 Alfred P. Sloan Foundation Fellow, supported in part by the U. S. Air Force Office of Scientific Research and by a Shell Grant at the Courant Institute of Mathematical Sciences. 
The field $\phi(x, t)$ is also space time translation covariant [3]-[4]. If $a=(\alpha, \tau) \in R^{2}$, there is a unitary operator

$$
U(a)=\exp (i H \tau-i P \alpha)
$$

such that

$$
\phi(x+\alpha, t+\tau)=U(a) \phi(x, t) U(a)^{*} .
$$

The transformation (4)-(5) uniquely determines a ${ }^{*}$-isomorphism

$$
\sigma_{a}: \mathfrak{A}(B) \rightarrow \mathfrak{A}(ß+a)=U(a) \mathfrak{A}(\beta) U(a)^{*}
$$

for each $\mathbb{B}$, and $\sigma_{a}$ extends uniquely to a ${ }^{*}$-automorphism of $\mathfrak{A}$.

II. Relativistic covariance. Covariance of the field under Lorentz transformation is a usual axiom of quantum field theory. We prove that this axiom holds for the $\lambda\left(\phi^{4}\right)_{2}$ theory of [1]-[4]. To formulate Lorentz covariance, let $\beta$ denote the restricted Poincare group of transformations. An element $\left\{a, \Lambda_{\beta}\right\} \in \mathcal{P}$ is defined by its action on $R^{2}$,

$$
\begin{aligned}
&\left\{a, \Lambda_{\beta}\right\}(x, t)=(x(\cosh \beta)+t(\sinh \beta)+\alpha, \\
&x(\sinh \beta)+t(\cosh \beta)+\tau) .
\end{aligned}
$$

Hence $P$ is the semidirect product of the space-time translations $a$ with the pure Lorentz transformations $\Lambda_{\beta}$ (corresponding to velocity boosts $\tanh \beta$ ).

THEOREM. For each $\left\{a, \Lambda_{\beta}\right\} \in \mathcal{P}$ and each bounded set $\beta \subset R^{2}$, there is a unitary operator $U$ so that for all $(x, t) \in \mathbb{B}$,

$$
\phi\left(\left\{a, \Lambda_{\beta}\right\}(x, t)\right)=U \phi(x, t) U^{*},
$$

as an equality between operator valued distributions.

CoROLlaRy 1. There is a representation $\sigma_{\left\{a, \Delta_{\beta}\right\}}$ of $\odot$ by $*_{\text {-automor- }}$ phisms of $\mathfrak{A}$ such that

$$
\sigma_{\left\{a, \Lambda_{\beta}\right\}}: \mathfrak{A}(\mathbb{B}) \rightarrow \mathfrak{A}\left(\left\{a, \Lambda_{\beta}\right\} \mathbb{B}\right) .
$$

We first establish the theorem for pure Lorentz transformations $\left\{0, \Lambda_{\beta}\right\}$ and compact sets $\beta_{1}$ in the quadrant $\{x>|t|\}$. Given $\beta_{0}>0$, we construct a unitary group $U(\beta)=\exp (i M \beta)$ satisfying (8) for $|\beta|<\beta_{0}$. Our choice of a selfadjoint local generator $M$ is motivated by familiar formal expressions in the physics literature. It is technically convenient to have $0 \leqq M$, which is possible only if $B_{1} \subset\{x>|t|\}$.

The key step in proving (8) is to show that $\phi(x, t)$ of $(5)$ satisfies the partial differential equation 


$$
\left\{t \frac{\partial}{\partial x}+x \frac{\partial}{\partial t}\right\} \phi(x, t)=[i M, \phi(x, t)]
$$

We obtain (10) from a lengthy series of estimates which allow us to compute $[i M, \phi]$ on a dense subset of $\mathfrak{H C} \times \mathcal{H C}$. We then integrate (10) to obtain (8) for our special case. The general case follows, since a Poincaré transformation from an arbitrary bounded set $B \subset R^{2}$ can be expressed as a product of space-time translations (6) and a pure Lorentz transformation from a compact set $B_{1} \subset\{x>|t|\}$.

Corollary 1 , together with the results of [3] asserts the following:

Corollary 2. The algebras $\mathfrak{Y}(ß)$ and $\mathfrak{A}$ of the $\lambda\left(\phi^{4}\right)_{2}$ quantum field theory satisfy all of the Haag-Kastler axioms for a quantum field theory [5].

\section{REFERENCES}

1. J. Glimm and A. Jaffe, $A \lambda \phi^{4}$ quantum field theory without cutoffs. I, Phys. Rev. 176 (1968), 1945-1951.

2. - Singular perturbations of self adjoint operators, Comm. Pure Appl. Math. 22 (1969), 401-414.

3. - The $\lambda\left(\phi^{4}\right)_{2}$ quantum field theory without cutoffs. II: The field operators and the approximate vacuum, Ann. of Math. (2) 91 (1970), 362-401.

4. - The $\lambda\left(\phi^{4}\right)_{2}$ quantum field theory without cutoffs. III: The physical vacuum, Acta. Math. (to appear).

5. R. Haag and D. Kastler, An algebraic approach to quantum field theory, J. Mathematical Phys. 5 (1964), 848-861. MR 29 \#3144.

Massachusetts Institute of Technology, Cambridge, Massachusetts 02139

Harvard University, Cambridge, Massachusetts 02138 